\title{
Performance Analysis of Space Information Networks with Backbone Satellite Relaying for Vehicular Networks
}

\author{
Jian Jiao, Houlian Gao, Shaohua Wu, and Qinyu Zhang \\ Communication Engineering Research Centre, Harbin Institute of Technology, Shenzhen, Guangdong, China \\ Correspondence should be addressed to Shaohua Wu; hitwush@hit.edu.cn
}

Received 9 September 2017; Accepted 15 November 2017; Published 10 December 2017

Academic Editor: Tao Han

Copyright (C) 2017 Jian Jiao et al. This is an open access article distributed under the Creative Commons Attribution License, which permits unrestricted use, distribution, and reproduction in any medium, provided the original work is properly cited.

\begin{abstract}
Space Information Network (SIN) with backbone satellites relaying for vehicular network (VN) communications is regarded as an effective strategy to provide diverse vehicular services in a seamless, efficient, and cost-effective manner in rural areas and highways. In this paper, we investigate the performance of SIN return channel cooperative communications via an amplify-andforward (AF) backbone satellite relaying for VN communications, where we assume that both of the source-destination and relaydestination links undergo Shadowed-Rician fading and the source-relay link follows Rician fading, respectively. In this SIN-assisted $\mathrm{VN}$ communication scenario, we first obtain the approximate statistical distributions of the equivalent end-to-end signal-to-noise ratio (SNR) of the system. Then, we derive the closed-form expressions to efficiently evaluate the average symbol error rate (ASER) of the system. Furthermore, the ASER expressions are taking into account the effect of satellite perturbation of the backbone relaying satellite, which reveal the accumulated error of the antenna pointing error. Finally, simulation results are provided to verify the accuracy of our theoretical analysis and show the impact of various parameters on the system performance.
\end{abstract}

\section{Introduction}

Nowadays, the connected vehicles paradigm is to form a vehicular network (VN) to communicate with the surrounding environment and the $\mathrm{VN}$ plays a vital role in the next generation intelligent transportation system (ITS) [1]. Generally, the long-term evolution (LTE) can provide reliable access to the Internet for $\mathrm{VN}$ communications in the urban areas. However, LTE network has poor coverage in rural areas and highways due to the costly network infrastructure $[2,3]$. Moreover, the high mobility of vehicles can suffer from frequent handovers as the networks become even denser.

Space Information Network (SIN) is regarded as an effective strategy to provide diverse vehicular services in a seamless, efficient, and cost-effective manner in rural areas and highways. For instance, satellites and high altitude platforms (HAPs) in SIN can help achieve ubiquitous coverage in rural areas. Further, they can provide road information and transport information to assist ITS, entertainment services dissemination as relays, and relieve the demands on terrestrial networks through data offloading [4].
The return channels of the low/medium Earth orbit (L/MEO) satellites are unstable and discontinuous intrinsically to the ground-based stations and vehicles, which limit the throughput as well as the delay sensitive services of SIN-assisted VN communications. Recently, high throughput backbone satellites (such as the Ka/Q/V-band geostationary Earth orbit (GEO) satellites) relaying for SIN communications are regarded as an effective strategy to improve the continuity of return channels as well as the throughput performance.

Theoretically, three GEO satellites which are $120^{\circ}$ apart in the SIN backbone networks can provide coverage of the space between Earth ground and GEO orbit and achieve high-speed data relay through the intersatellite and satellite-terrestrial millimeter/terahertz/laser links.

With the development of high throughput satellites (HTS), several GEO HTS can establish the backbone network of SIN, where the backbone HTS relaying for SIN-assisted VN is able to provide a global seamless broadband transmission by developing the intersatellite links. People believe that the SIN will enable a "terabit data rate capacity" broadband 
access, which was previously possible only with fiber-optic links, and offer the access availability of "anywhere and anytime" inherent to the satellites [5]. Furthermore, the SIN will be a significant enabling factor as well as an important component of the upcoming 5th-generation (5G) networks [6].

Therefore, considering the backbone HTS relaying communication undergoes the large-scale and complex SIN dualhop channel properties, such as rain attenuation [7], solar scintillation [8], perturbation factors [9], and interference [10-13], this paper investigates the performance of SIN return channel cooperative communications via an amplify-andforward (AF) backbone satellite relaying for VN communications.

1.1. Background and Motivation. In our SIN communication scenario, space-based nodes (i.e., source nodes, like space mission explorers, orbiters and landers, space stations, spacecraft, manned and unmanned aircraft, etc.) can establish cooperative communications via an AF backbone HTS relaying.

Recently, SINs have attracted considerable research interest, and substantial effort has been devoted to investigating the performance of the research works of the hybrid satelliteterrestrial cooperative/relay networks (HSTC/RNs) by analyzing the complex multihop channel models. For that, by applying maximal ratio combining (MRC) at the destination, $[14,15]$ studied the outage probability (OP) performance of HSTCNs with an AF relaying protocol. In [16], the decodeand-forward (DF) relaying protocols for HSTCNs was investigated. Further, with the help of the moment generating function (MGF), [14, 15, 17] have presented the analytical expression of average symbol error rate (ASER) for HSTCNs with an AF relaying protocol. Besides, the performance of optimal selection algorithm of multiple relays for HSTRNs was presented in $[18,19]$.

Moreover, to achieve higher system capacity and energy efficiency, multiantenna technique was investigated in [2022 ], and HTS with Ka/Q/V-band frequency with multiple antennas have attracted significant attention [23]. Authors in [24-31] investigate the performance of relay-based multiple antenna HSTC/RNs, since relay transmissions can effectively improve the throughput and the coverage of satellite communications. Further, the cognitive radio (CR) needs to be investigated since the HTS already suffer from spectrum scarcity in Ka band [32].

Besides, the SINs backbone GEO satellites are subjected to various satellite perturbation forces (e.g., Earth oblateness perturbation, third-body gravitational perturbation, atmospheric perturbation, and solar perturbation), which leads to position drift and result in the beam center of the ground station antenna unfocused [33]. The accumulated error of the antenna pointing error will cause the satellite elevation error, which may deteriorate the signal-to-noise ratio (SNR), decrease link margin [34] and bit error rate (BER) [35], and so forth. To the best of our knowledge, this is the first work on GEO satellite perturbation that reveals the effect of satellite elevation error for SIN backbone satellite relaying.
1.2. Contributions and Novelty. In this paper, we investigate the performance of SIN return channel cooperative communications via an AF backbone satellite relaying for $\mathrm{VN}$, where both of the source-destination and relay-destination links undergo Shadowed-Rician fading, and the source-relay link follows Rician fading, respectively. By applying MRC at the destination, the equivalent end-to-end SNR of the system is first obtained, and then analytical expressions as well as the satellite perturbation effect are derived to evaluate the system performance. The detailed contributions of this paper are outlined as follows:

(i) The system model of SIN return channel cooperative communications via an AF backbone satellite relaying for $\mathrm{VN}$ is first built, and we present a new analytical expression for the approximate statistical distributions of the equivalent end-to-end SNR of system (7).

(ii) To gain further insight, the effect of the satellite perturbation of the relaying GEO satellite is considered for the first time, which reveals the accumulated error of the antenna pointing error leads to the satellite elevation error. And the accumulated satellite elevation error is taking into account the derivation of the ASER expression.

(iii) The closed-form expression for the end-to-end ASER (31) is derived, which can efficiently evaluate the system performance. Moreover, simulation results prove the rationality of our theoretical analysis.

Notations. $X-Y$ describes the link from node $X$ to node $Y$. $X-Y-Z$ represents the dual-hop link from node $X$ to node $Z$ through relay node $Y$. E[.] denotes the expectation operator. $N\left(\mu, \sigma^{2}\right)$ denotes a complex Gaussian distribution with mean $\mu$ and variance $\sigma^{2} \cdot \exp (\cdot)$ represents the exponential function. $M_{\lambda}(\cdot)$ denotes the moment generating function (MGF) of $\gamma$. $f_{x}(\cdot)$ and $F_{x}(\cdot)$ denote the probability distribution function (PDF) and cumulative distribution function (CDF) of $x$, respectively. ${ }_{1} F_{1}(a ; b ; c)$ represents the confluent hypergeometric function of first kind [36, Eq. (9.210.1)]. $F(a, b ; c ; d)$ is Gauss hypergeometric function [36, Eq. (9.100)], and $K_{n}(\cdot)$ represents the modified Bessel function of the second kind with order $n$ [36, Eq. (8.446)]. $M_{a, b}(\cdot)$ is the Whittaker function defined as [36, Eq. (9.220.2)].

\section{System Model}

Our system model of the SIN return channel cooperative communications via an AF GEO HTS relaying for VN is considered as shown in Figure 1, where a source node $(S)$, that is, space node, communicates with a terrestrial destination $(D)$ via a GEO HTS relay $(R)$ and $h_{0}, h_{1}$, and $h_{2}$ are the channel gains of the $S-R, S-D$, and $R-D$ links, respectively.

The space node $S$ is generally on the stratosphere layer and above, and $R$ is a GEO HTS in our SIN communication scenario. In the $S-R$ link, since the line of sight (LOS) signal is much stronger than the others, which is different from terrestrial networks, the channel gain $h_{0}$ of the $S-R$ link is considered as a Rician fading with additive white Gaussian noise (AWGN) [37,38]. On the other hand, the channel gains 


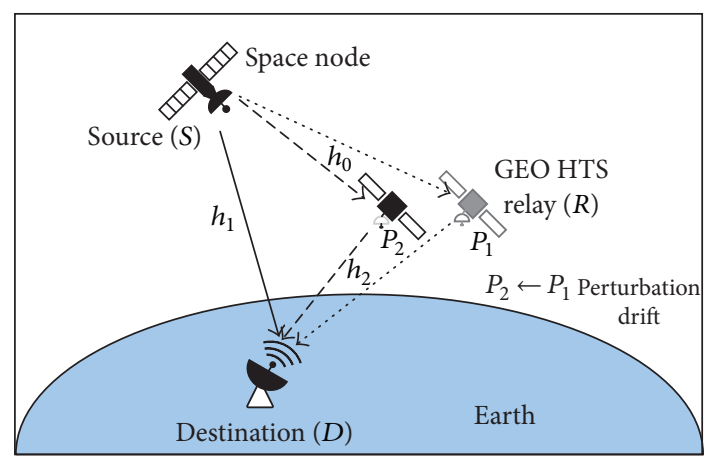

$\longrightarrow$ Direct link

$-\rightarrow$ Relay link

…> Perturbation relay link

FIGURE 1: The proposed system model of SIN return channel cooperative communications via an AF GEO HTS relaying for $\mathrm{VN}$, where each node is equipped with a single antenna, the relay point $P_{1}$ with an arrow point to $P_{2}$ shows the satellite drift effect caused by the satellite perturbation.

$h_{1}$ and $h_{2}$ of satellite-terrestrial links $S-D$ and $R-D$ are usually modeled by Shadowed-Rician fading distribution [14-16, 24-27, 35]. It approaches the LOS communication using the Rician fading, whereas the amplitude is Nakagami-m distributed [39], and it sufficiently agrees with experimental data and is computationally less complex than other land mobile satellite channel models.

As illustrated in Figure 1, in such a backbone GEO HTS relaying SIN-assisted VN system, the communication occurs during two time phases. In the first time phase, the space node $S$ broadcasts its signal to the relay $R$ and the destination $D$, where $h_{0}$ and $h_{1}$ are the channel gains of the $S-R$ and $S-D$ links, respectively. The received signals at the relay $y_{0}$ and the destination $y_{1}$ from $S$ are given by

$$
\begin{aligned}
& y_{0}=\sqrt{E_{1}} h_{0} x+n_{0}, \\
& y_{1}=\sqrt{E_{1}} h_{1} x+n_{1},
\end{aligned}
$$

where $x$ is the transmitted signal with unit power, $E_{1}$ is the transmitted power at $S$, and $n_{0}$ and $n_{1}$ are the AWGN of $S-R$ and $S-D$ links with zero mean and variance $\sigma_{0}^{2}$ and $\sigma_{1}^{2}$, respectively.

During the second time phase, $R$ first amplifies the received signal $y_{0}$ by an amplifying factor $G$ and then forwards it to $D$ through $R-D$ link of which the channel gain is $h_{2}$, and the received signal at the destination $y_{2}$ is given by

$$
\begin{aligned}
y_{2} & =\sqrt{E_{2}} G h_{2} y_{0}+n_{2} \\
& =G \sqrt{E_{1} E_{2}} h_{2} h_{0} x+G \sqrt{E_{2}} h_{2} n_{0}+n_{2},
\end{aligned}
$$

where $E_{2}$ is the transmit power at $R$ and $n_{2}$ is in AWGN at $D$ obeying $n_{2} \sim N\left(0, \sigma_{2}^{2}\right)$.
Assuming that perfect channel state information (CSI) is available at $D$ and $R$ and MRC is applied at the destination, thus, the end-to-end SNR at $D$ can be expressed as

$$
\gamma_{e 2 e}=\gamma_{1}+\gamma_{02}
$$

where $\gamma_{1}$ is the SNR of $S-D$ link and $\gamma_{02}$ is the SNR of $S-R-D$ link. From (2), we have

$$
\gamma_{1}=\frac{E_{1}\left|h_{1}\right|^{2}}{\sigma_{1}^{2}}=\rho_{1}\left|h_{1}\right|^{2} .
$$

From (3), $\gamma_{02}$ can be expressed as

$$
\gamma_{02}=\frac{E_{1}\left|h_{0}\right|^{2} / \sigma_{0}^{2} \cdot E_{2}\left|h_{2}\right|^{2} / \sigma_{2}^{2}}{E_{2}\left|h_{2}\right|^{2} / \sigma_{2}^{2}+1 / G^{2} \sigma_{0}^{2}}=\frac{\gamma_{0} \gamma_{2}}{\gamma_{2}+C}
$$

where $C=1 /\left(G \sigma_{0}\right)^{2}$ and $\gamma_{0}=E_{1}\left|h_{0}\right|^{2} / \sigma_{0}^{2}=\rho_{0}\left|h_{0}\right|^{2}$ and $\gamma_{2}=$ $E_{2}\left|h_{2}\right|^{2} / \sigma_{2}^{2}=\rho_{2}\left|h_{2}\right|^{2}$ denote the SNR of $S-R$ and $R-D$ link, respectively. Thus, (4) can be rewritten as

$$
\gamma_{e 2 e}=\frac{\gamma_{0} \gamma_{2}}{\gamma_{2}+C}+\rho_{1}\left|h_{1}\right|^{2} .
$$

2.1. Satellite Perturbation. In this paper, the GEO HTS satellite is considered as backbone relaying node for SIN-assisted $\mathrm{VN}$ communications to enhance the continuity as well as the throughput of the return channel. This is the first work to analyze the performance of cooperative communication for SIN dual-hop channel properties. To gain further insight, the effect of the satellite perturbation of the GEO HTS is analyzed, which reveals that the accumulated error of the antenna pointing error leads to the satellite elevation error.

2.1.1. Principle and Law of Satellite Perturbation Drift. The satellite is always subjected to a variety of perturbation forces, especially to the GEO satellites, which will lead to perturbation drift and accumulate the antenna pointing error. The satellite perturbation forces include the Earth oblateness perturbation [40, 41], the third-body attraction perturbation [42] such as lunisolar gravitational perturbation [43], the solar radiation pressure perturbation [44, 45], and the atmospheric drag perturbation.

The Earth oblateness perturbation is caused by the facts that the Earth is not an ideal sphere and it has uneven internal density distribution. It affects the long-term change of the right ascension of ascending node (RAAN) $r$ and argument of perigee $\omega$ of satellite orbit. The lunisolar gravitational perturbation can reduce the satellite orbit radius and may increase the orbital inclination, while the semimajor axis changes with half-day cycle. On the contrary, the solar radiation pressure perturbation mainly affects the orbital eccentricity, which directly determines the satellite center distance and the satellite height.

2.1.2. Satellite Elevation Error. The diagram of antenna pointing error is shown in Figure 2, and let $\theta=\theta_{e}+\theta_{c}$ denote the elevation of a GEO HTS, where $\theta_{e}$ represents the elevation 


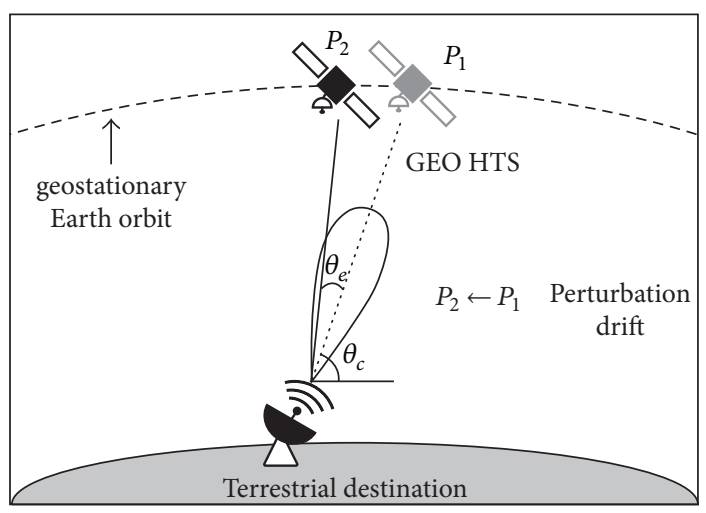

Figure 2: The diagram of antenna pointing error caused by satellite perturbation, $P_{1}$ means the satellite position located in antenna beam center, and $P_{2}$ represents the satellite position offsets from antenna beam center.

error, which is the angle between centerline of antenna beam pointed to $P_{1}$ and the real LOS channel between the actual position $P_{2}$ of the satellite to the destination. $\theta_{c}$ denotes the satellite elevation angle if the GEO satellite is not affected by the satellite perturbation. Considering the drift caused by satellite perturbation in the eastwest and northsouth directions, the elevation of satellite $\theta$ can be calculated by [46]

$$
\begin{aligned}
\theta & =\arctan \left[\frac{\sin \Delta \varphi \sin \phi+\cos \Delta \varphi \cos \phi \cos (\lambda+\Delta \lambda)-0.151}{\cos \{\arcsin [\sin \Delta \varphi \sin \phi+\cos \Delta \varphi \cos \phi \cos (\lambda+\Delta \lambda)]\}}\right], \\
\theta_{c} & =\arctan \left[\frac{\cos \phi \cos \lambda-0.151}{\cos [\arcsin (\cos \phi \cos \lambda)]}\right]_{\Delta \varphi=0},
\end{aligned}
$$

where $\phi$ is the latitude of the destination, $\lambda=\lambda_{\text {sat }}-\lambda_{\text {des }}$ is the longitude difference between the subsatellite point and the ground station, $\lambda_{\text {sat }}$ represents the longitude of subsatellite point, $\lambda_{\text {des }}$ is the longitude of destination, $\Delta \varphi$ is the drift of satellite in the northsouth direction, and $\Delta \lambda$ is the drift of satellite in the eastwest direction. Therefore, the elevation error $\theta_{e}$ can be calculated as $\theta_{e}=\theta-\theta_{c}$.

In general, $\lambda_{\text {des }}$ can be calculated by the six elements of the satellite orbit [47]. When the satellite orbit is elliptical, the longitude $\left(L_{\text {lon }}\right)$ and the latitude $\left(L_{\text {lat }}\right)$ of the subsatellite point can be expressed as

$$
\begin{aligned}
& L_{\text {lon }}=r+\arctan \{\cos i \\
& \left.\cdot \tan \left[\omega+2 \arctan \left(\sqrt{\frac{1+e}{1-e}} \tan \frac{E}{2}\right)\right]\right\}-\omega_{e}(t \\
& \left.-t_{N}\right) \\
& L_{\text {lat }}=\arcsin \{\sin i \\
& \left.\cdot \sin \left[\omega+2 \arctan \left(\sqrt{\frac{1+e}{1-e}} \tan \frac{E}{2}\right)\right]\right\},
\end{aligned}
$$

where $\omega_{e}$ is the average angular velocity of Earth, $t$ is the time, $t_{N}$ is the time when the satellite passes the ascending node, $E$ is the eccentric anomaly, $i$ is the inclination of satellite orbit, and $e$ is eccentricity. When the satellite orbit is a circle, (9) can be simplified as

$$
\begin{aligned}
L_{\text {lon }}= & r+\arctan \left\{\cos i \tan \left[\omega+\frac{2 \pi}{T}(t-\tau)\right]\right\} \\
& -\omega_{e}\left(t-\tau+\frac{T \omega}{2 \pi}\right) \\
L_{\text {lat }}= & \arcsin \left\{\sin i \sin \left[\omega+\frac{2 \pi}{T}(t-\tau)\right]\right\},
\end{aligned}
$$

where $\tau$ is the time when the satellite perigee passes.

Therefore, from (9) and (10), it is worth noting that the subsatellite point is related to the parameters $r, i, \omega$, and $\tau$. If these variables have been affected by the satellite perturbation, the subsatellite point will have $\operatorname{drift}$. Let $L_{p}^{\text {lon }}$ and $L_{p}^{\text {lat }}$ represent the longitude and latitude of subsatellite point considering the effect of satellite perturbation, respectively, and $L_{n p}^{\text {lon }}$ and $L_{n p}^{\text {lat }}$ denote the longitude and latitude of unperturbed subsatellite point, respectively. It is clear that the drift of subsatellite point affected by satellite perturbation causes the elevation error of satellite, and we have

$$
\begin{aligned}
& \Delta \varphi=L_{p}^{\text {lon }}-L_{n p}^{\text {lon }} \\
& \Delta \lambda=L_{p}^{\text {lat }}-L_{n p}^{\text {lat }} .
\end{aligned}
$$




\subsection{Channel Model}

2.2.1. $S-R$ Link. As the $S-R$ link is modeled by using the Rician fading distribution, the probability distribution function (PDF) of $\gamma_{0}$ is given by [48]

$$
\begin{aligned}
f_{\gamma_{0}}(x)= & \frac{(K+1)}{\rho_{0} \Omega} \exp \left(-\frac{x(K+1)}{\rho_{0} \Omega}-K\right) \\
& \cdot I_{0}\left(2 \sqrt{\frac{K(K+1) x}{\rho_{0} \Omega}}\right),
\end{aligned}
$$

where $\Omega$ is the average power of received signal at $R$ and $\Omega=\left(A^{2}+2 \sigma^{2}\right) / 2$ and $K=A^{2} /\left(2 \sigma^{2}\right)$ are known as the Rician factor, $A$ is the amplitude of LOS signal, $\sigma^{2}$ is the average power of multipath component, and $I_{0}(\cdot)$ is the modified Bessel function of the first kind with order zero.

Then, the cumulative distribution function (CDF) of $\gamma_{0}$ can be expressed as

$$
F_{\gamma_{0}}(x)=1-Q_{1}\left(\sqrt{2 K}, \sqrt{\frac{2(K+1) x}{\rho_{0} \Omega}}\right),
$$

where $Q_{M}(\cdot, \cdot)$ is the Marcum $Q$-function of the $M$ th order, which is defined as

$$
\begin{aligned}
& Q_{M}(a, b) \\
& \quad=\int_{b}^{\infty} x\left(\frac{x}{a}\right)^{M-1} \exp \left(-\frac{x^{2}+a^{2}}{2}\right) I_{M-1}(a x) d x .
\end{aligned}
$$

Moreover, the relationship between the Rician factor $K$ of the Rician fading $S-R$ link (12) and the satellite elevation $\left(\theta_{c}\right)$ was simulated in [49] through a large number of experiments. Then the relationship between the Rician factor and the satellite elevation $\left(\theta_{c} \in\left[10^{\circ}, 90^{\circ}\right]\right)$ was fitted as an empirical formula

$$
K\left(\theta_{c}\right)=K_{0}+K_{1}\left(\theta_{c}\right)+K_{2}\left(\theta_{c}\right)
$$

where $K_{0}, K_{1}$, and $K_{2}$ are empirical constant and $K_{0}=2.731$, $K_{1}=-1.074 \times 10^{-1}$, and $K_{2}=2.771 \times 10^{-3}$, respectively. Considering the effect of satellite perturbation, (15) can be rewritten as

$$
\begin{aligned}
K\left(\theta_{c}, \theta_{e}, t\right)= & K_{0}+K_{1}\left(\theta_{c}+\theta_{e} \sin t\right) \\
& +K_{2}\left(\theta_{c}+\theta_{e} \sin t\right),
\end{aligned}
$$

where $\theta_{e} \sin t$ indicates the elevation error $\theta_{e}$ affected by the satellite perturbation at time $t$.

2.2.2. $S-D$ and $R-D$ Links. The $S-D$ link is usually modeled as a composite fading distribution to describe the amplitude fluctuation of the signal envelope. Considering the tradeoffs between accuracy and computational complexity, the satellite-destination $S-D$ link and the relay-destination
$R-D$ link are modeled by using the Shadowed-Rician fading distribution [11-13] in our SIN-assisted VN system.

Let $\gamma_{1}$ and $\gamma_{2}$ denote the SNR of the $S-D$ and $R-D$ links, respectively. The PDF of $\gamma_{i}(i=1,2)$ is given by [39]

$$
f_{\gamma_{i}(x)}=\frac{\alpha_{i}}{\rho_{i}} \exp \left(-\frac{\beta_{i} x}{\rho_{i}}\right) \cdot{ }_{1} F_{1}\left(m_{i} ; 1 ; \frac{\delta_{i}}{\rho_{i}} x\right),
$$

where

$$
\begin{aligned}
& \alpha_{i}=\frac{\left(2 b_{i} m_{i} /\left(2 b_{i} m_{i}+\Omega_{i}\right)\right)^{m_{i}}}{2 b_{i}}, \\
& \beta_{i}=\frac{1}{2 b_{i}}, \\
& \delta_{i}=\frac{0.5 \Omega_{i}}{\left(2 b_{i}^{2} m_{i}+b_{i} \Omega_{i}\right)},
\end{aligned}
$$

where ${ }_{1} F_{1}(\cdot ; \cdot ;)$ is the confluent hypergeometric function of first kind [36, Eq. (9.210.1)]. Moreover, $\Omega_{i}$ and $2 b_{i}$ are the average power of the LOS and the multipath components, respectively, and $m_{i}\left(m_{i} \in[0, \infty)\right)$ is the fading severity parameter.

Recall the definition of ${ }_{1} F_{1}(\cdot ; \cdot ; \cdot)$, and we have

$$
{ }_{1} F_{1}\left(m_{i} ; 1 ; \frac{\delta_{i}}{\rho_{i}} x\right)=\sum_{n=0}^{\infty} \frac{\left(m_{i}\right)_{n}}{(1)_{n}} \frac{1}{n !}\left(\frac{\delta_{i}}{\rho_{i}} x\right)^{n} .
$$

For the analytical tractability, we retain our focus in the case when the channel severity parameters take integer values in the rest of this paper; that is, $m_{i} \in \mathrm{N}$. Hence, with the aid of [50, Eq. (07.20.03.0009.01), Eq. (07.02.03.0014.01)], (19) becomes

$$
\begin{aligned}
{ }_{1} F_{1} & \left(m_{i} ; 1 ; \frac{\delta_{i}}{\rho_{i}} x\right) \\
& =\exp \left(\frac{\delta_{i}}{\rho_{i}} x\right) \sum_{n=0}^{m_{i}-1} \frac{(-1)^{n}\left(1-m_{i}\right)_{n}}{(n !)^{2}}\left(\frac{\delta_{i}}{\rho_{i}} x\right)^{n},
\end{aligned}
$$

where $(z)_{n}=\Gamma(z+n) / \Gamma(z)$ denotes the Pochhammer symbol with $n \in N$ [51, Eq. (6.1.22)].

To solve the three parameters $\left(\Omega_{i}, b_{i}\right.$, and $\left.m_{i}\right)$ in (18), we assume $\theta_{c i}$ is the elevation at GEO HTS $R$, when the center line of the receiving antenna beam in different link aims at R. $\theta_{e i} \sin t$ is the elevation error which affects the satellite perturbation. When $\theta_{c i} \in\left(20^{\circ}, 30^{\circ}\right), \Omega_{i}, b_{i}$, and $m_{i}$ can be calculated by the empirical formulas [39, Eq. (19)]. Therefore, 
considering the effect of satellite perturbation, $\Omega_{i}, b_{i}$, and $m_{i}$ can be calculated as follows:

$$
\begin{aligned}
b_{i}\left(\theta_{c i}, \theta_{e i}, t\right)= & -4.7943 \times 10^{-8}\left(\theta_{c i}+\theta_{e i} \sin t\right)^{3} \\
& +5.5784 \times 10^{-6}\left(\theta_{c i}+\theta_{e i} \sin t\right)^{2} \\
& -2.1344 \times 10^{-4}\left(\theta_{c i}+\theta_{e i} \sin t\right) \\
& +3.2710 \times 10^{-2}, \\
m_{i}\left(\theta_{c i}, \theta_{e i}, t\right)= & 6.3739 \times 10^{-5}\left(\theta_{c i}+\theta_{e i} \sin t\right)^{3} \\
& +5.8533 \times 10^{-4}\left(\theta_{c i}+\theta_{e i} \sin t\right)^{2} \\
& -1.5973 \times 10^{-4}\left(\theta_{c i}+\theta_{e i} \sin t\right) \\
& +3.5156, \\
\Omega_{i}\left(\theta_{c i}, \theta_{e i}, t\right)= & 1.4428 \times 10^{-5}\left(\theta_{c i}+\theta_{e i} \sin t\right)^{3} \\
& -2.3798 \times 10^{-3}\left(\theta_{c i}+\theta_{e i} \sin t\right)^{2} \\
& +1.2702 \times 10^{-1}\left(\theta_{c i}+\theta_{e i} \sin t\right) \\
& -1.4864 .
\end{aligned}
$$

\section{Performance Analysis}

In order to exactly measure the effect of satellite perturbation on the SIN return channel cooperative communications via an AF GEO HTS relaying, the important quality-ofservice (QoS) metric, that is, average symbol error probability (ASER), is analytically studied and evaluated in our proposed SIN-assisted VN systems.

Since MRC is applied at the destination, we derive the close-form expression by using MGF according to [52], where the ASER of an $M$-ary phase-shift keying (MPSK) modulated system is given by

$$
P_{\mathrm{MPSK}}=\frac{1}{\pi} \int_{0}^{\theta_{M}} M_{\gamma_{\text {eez }}}\left(\frac{g_{\mathrm{MPSK}}}{\sin ^{2} \theta}\right),
$$

where $\theta_{M}=\pi(M-1) / M$ and $g_{\mathrm{MPSK}}=\sin ^{2}(\pi / M)$. The MGF of instantaneous $\operatorname{SNR}(\gamma)$ is defined as

$$
M_{\gamma}=E_{\gamma}\left\{e^{-s \gamma}\right\}=\int_{0}^{\infty} e^{-s \gamma} f_{\gamma}(\gamma) d \gamma
$$

Considering $h_{0}, h_{1}$, and $h_{2}$ are independent and the relationship between $\gamma_{1}, \gamma_{02}$, and $\gamma_{e 2 e}$ is presented in (4), we can express $M_{e 2 e}(s)$ as

$$
M_{\gamma_{e 2 e}}(s)=M_{\gamma_{1}}(s) M_{\gamma_{02}}(s),
$$

where $M_{\gamma_{1}}(s)$ and $M_{\gamma_{02}}(s)$ are the MGF of $\gamma_{1}$ and $\gamma_{02}$.

In the following, we derive the expressions for $M_{\gamma_{1}}(s)$ and $M_{\gamma_{02}}(s)$. Then, we use (22) and (24) to obtain the ASER of our SIN backbone satellite relaying $\mathrm{VN}$ system.
3.1. MGF of the SNR for the S-D Link. By using the definition of MGF and substituting (17) into (23), we can evaluate the MGF of the $S-D$ link as follows

$$
\begin{aligned}
M_{\gamma_{1}}(s) & =\mathrm{E}\left\{e^{-s x}\right\} \\
& =\int_{0}^{\infty} e^{-s x} \frac{\alpha_{1}}{\rho_{1}} e^{-\left(\beta_{1} / \rho_{1}\right) x} \cdot{ }_{1} F_{1}\left(m_{1} ; 1 ; \frac{\delta_{1}}{\rho_{1}} x\right) d x \\
& =\frac{\alpha_{1}}{\rho_{1}} \int_{0}^{\infty} e^{-\left(\beta_{1} / \rho_{1}+s\right) x} \cdot{ }_{1} F_{1}\left(m_{1} ; 1 ; \frac{\delta_{1}}{\rho_{1}} x\right) d x
\end{aligned}
$$

By using [36, Eq. (7.621.4)], the result of (25) can be easily obtained as follows:

$$
M_{\gamma_{1}}(s)=\frac{\alpha_{1}}{\rho_{1} s+\beta_{1}} F\left(m_{1}, 1 ; 1 ; \frac{\delta_{1}}{\rho_{1} s+\beta_{1}}\right) .
$$

3.2. MGF of the SNR for the S-R-D Link. By substituting $\gamma_{02}$ in (6) into (23), we can evaluate the MGF of cooperative link as presented in (27). The proof is provided in the Appendix.

$$
\begin{aligned}
& M_{\gamma_{02}}(s)=\frac{u_{2}(s) \alpha_{2} e^{-K+u_{2}(s)+\left(\left(\beta_{2}-\delta_{2}\right) / \rho_{2}\right) u_{1}(s)}}{K \rho_{2}} \\
& \cdot \sum_{n=0}^{m_{2}-1} \frac{(-1)^{n}\left(1-m_{2}\right)_{n}}{(n !)^{2}}\left(\frac{\delta_{2}}{\rho_{2}}\right)^{n} \sum_{k=0}^{n}\left[-u_{1}(s)\right]^{n-k} \\
& \cdot\left[2\left(\frac{\varepsilon \rho_{2} u_{2}(s)}{\beta_{2}-\delta_{2}}\right)^{(k+1) / 2}\right. \\
& \cdot K_{k+1}\left(2 \sqrt{\frac{\varepsilon u_{2}(s)\left(\beta_{2}-\delta_{2}\right)}{\rho_{2}}}\right) \\
& \left.+2 \varepsilon\left(\frac{\varepsilon \rho_{2} u_{2}(s)}{\beta_{2}-\delta_{2}}\right)^{k / 2} K_{k}\left(2 \sqrt{\frac{\varepsilon u_{2}(s)\left(\beta_{2}-\delta_{2}\right)}{\rho_{2}}}\right)\right],
\end{aligned}
$$

where the definitions $u_{1}(s), u_{2}(s)$, and $\varepsilon$ are shown in (A.7).

3.3. Derivation of ASER. Based on the definition of ASER of an MPSK modulated system (i.e., (27) and (24)), (22) can be written as

$$
\begin{aligned}
& P_{\text {MPSK }}=\frac{1}{\pi} \int_{0}^{\theta_{M}} M_{\gamma_{\text {eee }}}\left(\frac{g_{\mathrm{MPSK}}}{\sin ^{2} \theta}\right) d \theta \\
& =E_{\gamma}\left[\frac{1}{\pi} \int_{0}^{\pi / 2} \exp \left(\frac{g_{\mathrm{MPSK}}}{\sin ^{2} \theta}\right) d \theta\right. \\
& \left.\quad+\frac{1}{\pi} \int_{\pi / 2}^{\theta_{M}} \exp \left(\frac{g_{\mathrm{MPSK}}}{\sin ^{2} \theta}\right) d \theta\right] \\
& =\frac{1}{\pi} \int_{0}^{\theta_{M}} M_{\gamma_{1}}\left(\frac{g_{\mathrm{MPSK}}}{\sin ^{2} \theta}\right) M_{\gamma_{02}}\left(\frac{g_{\mathrm{MPSK}}}{\sin ^{2} \theta}\right) d \theta .
\end{aligned}
$$


Alternatively, the following approximation of (28) can be used [37]:

$$
\begin{aligned}
P_{\text {MPSK }} & =\frac{1}{\pi} \int_{0}^{\theta_{M}} M_{\gamma_{1}}\left(\frac{g_{\text {MPSK }}}{\sin ^{2} \theta}\right) M_{\gamma_{02}}\left(\frac{g_{\text {MPSK }}}{\sin ^{2} \theta}\right) d \theta \\
& \approx \sum_{j=1}^{3} k_{j} M_{\gamma_{1}}\left(w_{j}\right) M_{\gamma_{02}}\left(w_{j}\right),
\end{aligned}
$$

where

$$
\begin{aligned}
& k_{1}=\frac{\theta_{M}}{2 \pi}-\frac{1}{6}, \\
& w_{1}=-g_{\mathrm{MPSK}}, \\
& k_{2}=\frac{1}{4}, \\
& w_{2}=-\frac{4}{3} g_{\mathrm{MPSK}}, \\
& k_{3}=\frac{\theta_{M}}{2 \pi}-\frac{1}{4}, \\
& w_{3}=-\frac{g_{\mathrm{MPSK}}}{\sin ^{2} \theta} .
\end{aligned}
$$

As shown in (26) and (27), the close forms of $M_{\gamma_{1}}(s)$ and $M_{\gamma_{02}}(s)$ have already been derived. By substituting these close forms into (29), we finally obtain the accurate closedform expression of the ASER of a space downlink cooperative transmission system with relay GEO satellite as shown in

$$
\begin{aligned}
P_{\text {MPSK }} & =\sum_{j=1}^{3} k_{j} \frac{\alpha_{1}}{\rho_{1} w_{j}+\beta_{1}} F\left(m_{1}, 1 ; 1 ; \frac{\delta_{1}}{\rho_{1} w_{j}+\beta_{1}}\right) \\
\cdot & \frac{u_{2}\left(w_{j}\right) \alpha_{2} e^{-K+u_{2}\left(w_{j}\right)+\left(\left(\beta_{2}-\delta_{2}\right) / \rho_{2}\right) u_{1}\left(w_{j}\right)}}{K \rho_{2}} \\
\times & \sum_{n=0}^{m_{2}-1} \frac{(-1)^{n}\left(1-m_{2}\right)_{n}}{(n !)^{2}}\left(\frac{\delta_{2}}{\rho_{2}}\right)^{n} \sum_{k=0}^{n}\left[-u_{1}\left(w_{j}\right)\right]^{n-k} \\
\times & \left.2\left(\frac{\varepsilon \rho_{2} u_{2}\left(w_{j}\right)}{\left.\beta_{2}-\delta_{2}\right)^{(k+1) / 2}}\right)\right] \\
\cdot & K_{k+1}\left(2 \sqrt{\frac{\varepsilon u_{2}\left(w_{j}\right)\left(\beta_{2}-\delta_{2}\right)}{\rho_{2}}}\right) \\
+ & 2 \varepsilon\left(\frac{\varepsilon \rho_{2} u_{2}\left(w_{j}\right)}{\beta_{2}-\delta_{2}}\right)^{k / 2} \\
& K_{k}\left(2 \sqrt{\frac{\varepsilon u_{2}\left(w_{j}\right)\left(\beta_{2}-\delta_{2}\right)}{\rho_{2}}}\right)
\end{aligned}
$$

TABLE 1: Initial position and velocity vector of GEO HTS and other system parameters.

\begin{tabular}{lc}
\hline Parameters & Description \\
\hline Earth gravity model & WGS84_EGM96.grv \\
Satellite mass & $1000 \mathrm{~kg}$ \\
Mass-area ratio of satellite & $0.1 \mathrm{~m}^{2} / \mathrm{kg}$ \\
Reflection coefficient of spacecraft & 1.2 \\
Solar radiation pressure model & Spherical \\
Third-body gravity & Sun, Moon \\
\hline
\end{tabular}

\section{Numerical Results}

This section gives the numerical results to demonstrate the validity of the theoretical analysis and the effect of satellite perturbation on the SIN return channel cooperative communications via a GEO HTS relaying.

We assume the node $S$ is a space node and its position is $L_{\text {lat }}=10^{\circ} \mathrm{N}$ and $L_{\text {lon }}=0^{\circ} \mathrm{W}$. The node $R$ is a GEO HTS and, for the initial position $(X, Y$, and $Z)$ and velocity vector $(\mathrm{V} x, \mathrm{~V} y$, and $\mathrm{V} z)$ in the Cartesian coordinate system, their initial values are $(X, Y$, and $Z)=(-32299.6,-27102.6,0) \mathrm{km}$ and $(\mathrm{V} x, \mathrm{~V} y$, and $\mathrm{V} z)=(1.97635,-2.35533,0) \mathrm{km} / \mathrm{sec}$. We adopt high precision orbit propagator (HPOP) model and the parameters of the various perturbations are shown in Table 1. The GEO HTS $R$ is mainly affected by the Earth gravity, the third-body gravity, and the solar radiation pressure perturbation [53]. In Table 1, the Earth gravity model [54], the solar radiation pressure perturbation, and the third-body gravity perturbation adopt general settings.

The elevation error affected by the satellite perturbation is shown in Figure 3, which is mainly considering the Earth nonspherical perturbation, the lunisolar gravitational perturbation, and the solar radiation pressure perturbation. The simulation duration is one lunar month and each step is 60 seconds.

As shown in Figure 3, the elevation error accumulates on the $R-D$ link which is about 1 degree after one lunar month. The elevation error fluctuates on the $S$ - $R$ link, where the range of fluctuation increases gradually and the simulation time accumulates, and the maximum of fluctuation is about 0.1 degrees at the end of the simulation.

We assume $E_{1}=E_{2}$ and $\rho_{0}=\rho_{1}=\rho_{2}=\rho$. In $S$ - $R$ and $R-D$ links, the elevations of $T_{X}$ ignoring satellite perturbation at nodes $R$ and $D$ are equal; that is, $\theta_{c 0}=\theta_{c 2}=\theta_{0}$. In order to simulate the influence of the elevation error caused by perturbation on the system ASER performance, the elevation error data is sampled at intervals of 12 hours due to the large amount of elevation error in Figure 3. The amplifying factor is $G=30$ and $\theta_{c 1}=60^{\circ}$ and QPSK modulation is implemented, and the rest of the simulation parameters are the same as above. The end-to-end ASER with satellite perturbation in the SIN return channel cooperative communications via an AF GEO HTS are shown in Figures 4, 5, and 6, respectively.

Figures 4 and 5 indicate the ASER performance is improving with the increasing of receiving $\operatorname{SNR} \rho$. As one can expect, with the increasing of $T_{X}$ and average power of received signal at node $R$ in the $S-R$ and $R-D$ links, the ASER performance is 


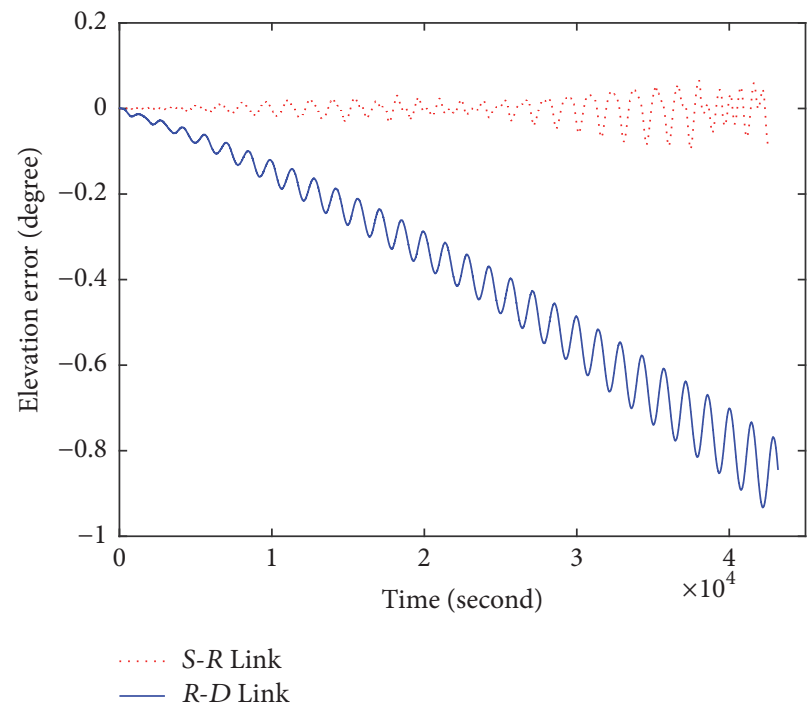

FIgURE 3: Elevation error caused by GEO satellite perturbation.

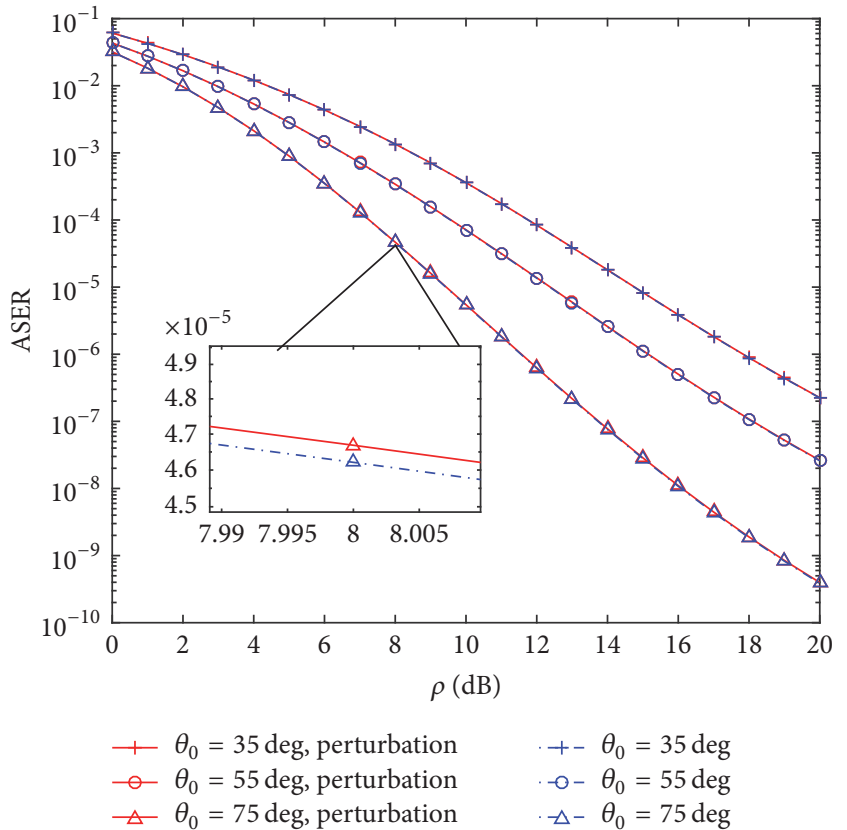

(a)
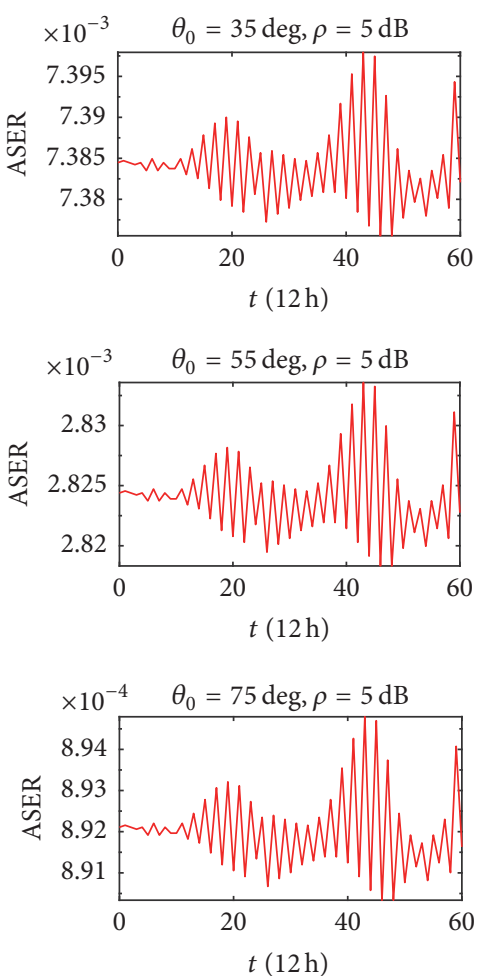

(b)

FIGURE 4: ASER with satellite perturbation versus various $\rho$; (b) shows the elevation error fluctuation and accumulation process on the ASER under different $\theta_{0}$, where $\rho=5 \mathrm{~dB}$ and $\Omega=5 \mathrm{~W}$.

improving. Comparing with the cases of ignoring the effect of the satellite perturbation, the ASER performance deteriorates a little due to the satellite perturbation.

Moreover, three subfigures in Figures 4 and 5 show the fluctuation and error accumulation process of the ASER with satellite perturbation with $\rho=5 \mathrm{~dB}$, under different $\theta_{0}$ and $\Omega$, respectively. In Figure 4 , when $\theta_{0}=35^{\circ}, 55^{\circ}$, and $75^{\circ}$, the fluctuation of ASER is the same, and the fluctuation ranges are $2.2379 e-5,1.5260 e-5$, and $4.4567 e-6$, respectively, and the fluctuation range becomes tighter and $\theta_{0}$ increases. Similar to Figure 5, when $\Omega=2 \mathrm{~W}, 5 \mathrm{~W}$, and $10 \mathrm{~W}$, the fluctuation of ASER is the same, and the fluctuation ranges are $2.9818 e-5$, $1.5260 e-5$, and $6.8832 e-6$, respectively, and the fluctuation range declines when $\Omega$ increases. To be clearer, we show the 


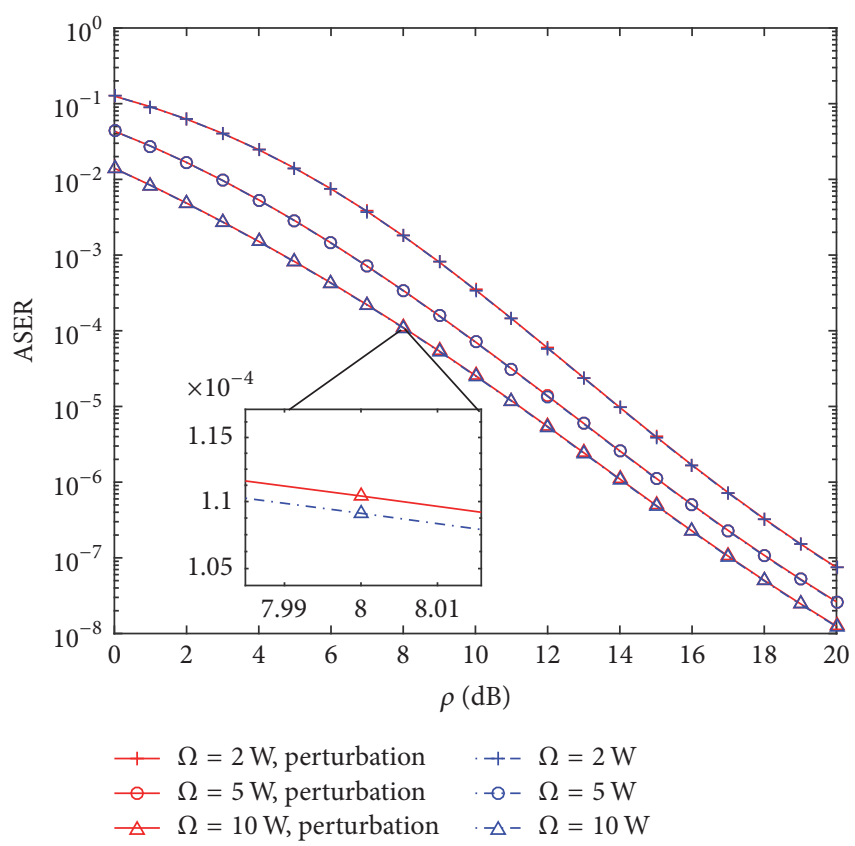

(a)
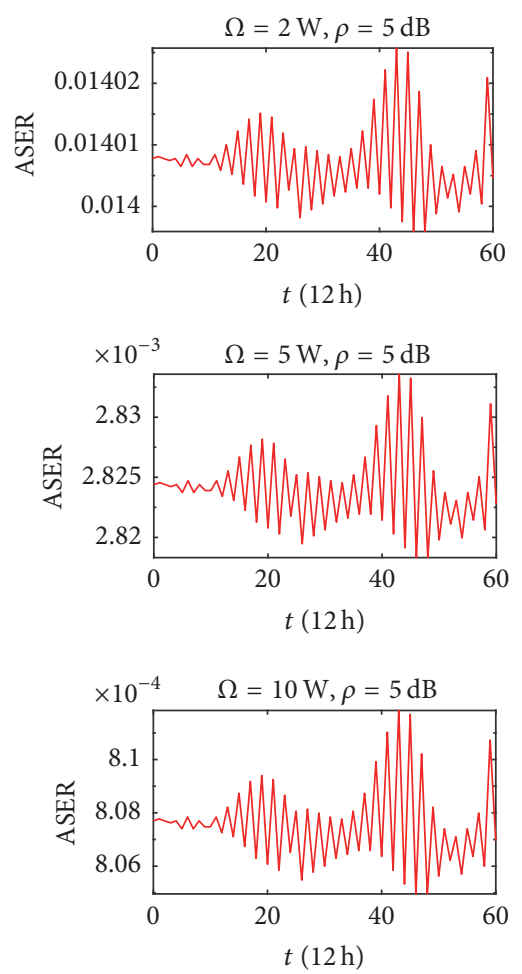

(b)

FIGURE 5: ASER with satellite perturbation versus various $\rho$; (b) shows the elevation error fluctuation and accumulation process on the ASER under different $\Omega$, where $\rho=5 \mathrm{~dB}$ and $\theta_{0}=55^{\circ}$.

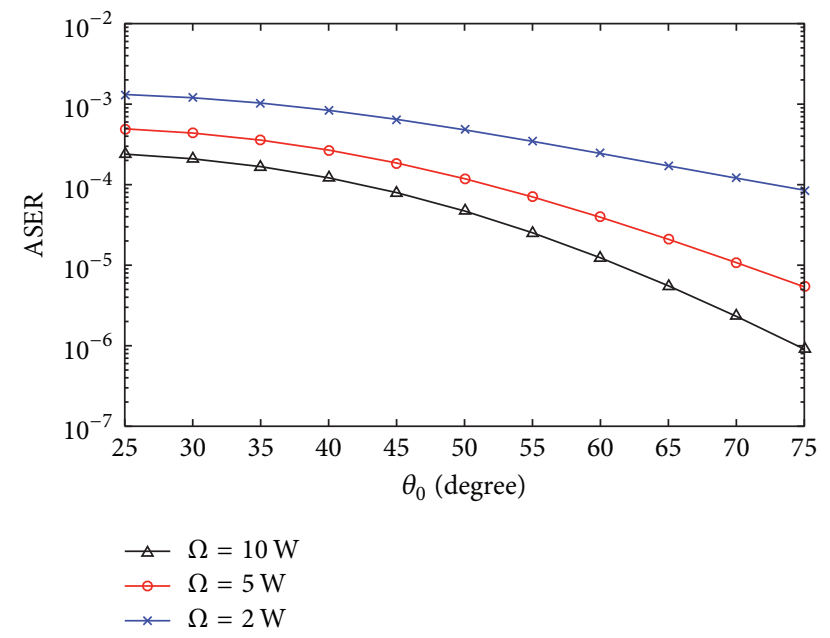

FIGURE 6: ASER performance versus various $\theta_{0}$ under $\rho=5 \mathrm{~dB}$.

ASER performance with the increasing of $\Omega$ and $\theta_{0}$ as in Figure 6.

\section{Conclusion}

In this paper, we investigate the ASER performance of SIN return channel cooperative communications via an AF GEO HTS relaying for $\mathrm{VN}$, where both of the $S-D$ and $R-D$ links undergo the Shadowed-Rician fading, and the $S$ - $R$ link follows Rician fading, respectively. By applying MRC at $D$, the equivalent end-to-end SNR of the system is first obtained, then the analytical expressions of ASER and the satellite perturbation effect are derived. The effect of the satellite perturbation of the relaying GEO satellite is considered for the first time, which reveals that the accumulated error of the antenna pointing error leads to the satellite elevation error. And the accumulated satellite elevation error is taking into account the derivation of the ASER expression. The closedform expression for the end-to-end ASER can efficiently evaluate the system performance, and simulation results prove the rationality of our theoretical analysis.

\section{Appendix}

\section{Derivation the MGF of the Cooperative Link}

By the definition of MGF, from (6) and (23), the MGF of the cooperative link can be evaluated:

$$
\begin{aligned}
M_{\gamma_{02}} & =E\left(e^{-s \gamma_{02}}\right) \\
& =\int_{0}^{\infty} e^{-s\left(\gamma_{0} \gamma_{2} /\left(\gamma_{2}+C\right)\right)} f_{\gamma_{02}}(\gamma) d \gamma \\
& =\int_{0}^{\infty} e^{-s(x y /(y+C))} f_{\gamma_{0}}(x) f_{\gamma_{2}}(y) d x d y .
\end{aligned}
$$


Considering $h_{0}$ and $h_{2}$ are independent, we first calculate the following integral of variable $x$ such that

$$
\begin{aligned}
L= & \int_{0}^{\infty} e^{-s(x y /(y+C))} f_{\gamma_{0}}(x) d x \\
= & \frac{K+1}{\rho_{0} \Omega} e^{-K} \int_{0}^{\infty} e^{-x\left(s y /(y+C)+(K+1) / \rho_{0} \Omega\right)} \\
& \cdot I_{0}\left(2 \sqrt{\frac{K(K+1) x}{\rho_{0} \Omega}}\right) d x .
\end{aligned}
$$

By using [36, Eq. (6.614.3)], we get

$$
\begin{aligned}
L= & \frac{K+1}{\rho_{0} \Omega} e^{-K} \\
& \cdot \frac{e^{(1 / 2)\left(\left(K(K+1) / \rho_{0} \Omega\right) /\left((K+1) / \rho_{0} \Omega+s y /(y+C)\right)\right)}}{\sqrt{\left((K+1) / \rho_{0} \Omega+s y /(y+C)\right)\left(K(K+1) / \rho_{0} \Omega\right)}} \\
& \cdot M_{-1 / 2,0}\left(\frac{K(K+1) / \rho_{0} \Omega}{(K+1) / \rho_{0} \Omega+s y /(y+C)}\right)
\end{aligned}
$$

where $M_{\lambda, \mu}(z)$ is the Whittaker functions defined as [36, Eq. $(9.220 .2)]$

$$
M_{\lambda, \mu}(z)=z^{\mu+1 / 2} e^{-z / 2} \Phi\left(\mu-\lambda+\frac{1}{2}, 2 \mu+1 ; z\right)
$$

where $\Phi(\alpha, \gamma ; z)$ is a second notation of confluent hypergeometric function, and when $\alpha=\gamma, \Phi(\alpha, \gamma ; z)$ has the relationship [36, Eq. (9.215)] as follows:

$$
\Phi(\alpha, \alpha ; z)=e^{z}
$$

Thus, (A.3) can be rewritten as

$$
\begin{aligned}
L= & \frac{(K+1) e^{-K}}{K+1+\rho_{0} \Omega s} \frac{y+C}{y+C(K+1) /\left(K+1+\rho_{0} \Omega s\right)} \\
& \cdot e^{\left(K(K+1) /\left(K+1+\rho_{0} \Omega s\right)\right)\left((y+C) /\left(y+C(K+1) /\left(K+1+\rho_{0} \Omega s\right)\right)\right)} .
\end{aligned}
$$

In order to make the derivation more clear, we define

$$
\begin{aligned}
\frac{C(K+1)}{K+1+\rho_{0} \Omega s} & =u_{1}(s), \\
\frac{K(K+1)}{K+1+\rho_{0} \Omega s} & =u_{2}(s), \\
C-u_{1} & =\varepsilon .
\end{aligned}
$$
as

After some algebra manipulations, we can rewrite (A.6)

$$
L=\frac{u_{2}(s) e^{-K+u_{2}(s)}}{K}\left(1+\frac{\varepsilon}{y+u_{1}(s)}\right) e^{\varepsilon u_{2}(s) /\left(y+u_{1}(s)\right)} .
$$

Now, we can rewrite $M_{\gamma_{02}}(s)$ as the integral of variable $y$

$$
\begin{aligned}
& M_{\gamma_{02}}(s)=\int_{0}^{\infty} \frac{u_{2}(s) e^{-K+u_{2}(s)}}{K}\left(1+\frac{\varepsilon}{y+u_{1}(s)}\right) \\
& \cdot e^{\varepsilon u_{2}(s) /\left(y+u_{1}(s)\right)} \frac{\alpha_{2}}{\rho_{2}} e^{-\left(\beta_{2} / \rho_{2}\right) y} \\
& \cdot{ }_{1} F_{1}\left(m_{2} ; 1 ; \frac{\delta_{2}}{\rho_{2}} y\right) d y \\
& =\frac{u_{2}(s) \alpha e_{2} e^{-K+u_{2}(s)}}{K \rho_{2}} \int_{0}^{\infty}\left(1+\frac{\varepsilon}{y+u_{1}(s)}\right) \\
& \cdot e^{\varepsilon u_{2}(s) /\left(y+u_{1}(s)\right)-\left(\beta_{2} / \rho_{2}\right) y} \cdot{ }_{1} F_{1}\left(m_{2} ; 1 ; \frac{\delta_{2}}{\rho_{2}} y\right) d y .
\end{aligned}
$$

Let $w(s)=y+u_{1}(s)$, and then we have $y=w(s)-u_{1}(s)$ and $d y=d w$. After some algebra manipulations, (A.9) can be written as the integral of variable $w$

$$
\begin{gathered}
M_{\gamma_{02}}(s)=\frac{u_{2}(s) \alpha_{2} e^{-K+u_{2}(s)+\left(\alpha_{2} / \rho_{2}\right) u_{1}(s)}}{K \rho_{2}} \\
\cdot \int_{0}^{\infty}\left(1+\frac{s}{w(s)}\right) e^{\varepsilon u_{2}(s) / w(s)-\left(\beta_{2} / \rho_{2}\right) w(s)} \\
\cdot{ }_{1} F_{1}\left(m_{2} ; 1 ; \frac{\delta_{2}}{\rho_{2}}\left(w(s)-u_{1}(s)\right)\right) d w .
\end{gathered}
$$

By substituting (20) into (A.10), we have

$$
\begin{aligned}
& M_{\gamma_{02}}(s)=\frac{u_{2}(s) \alpha_{2} e^{-K+u_{2}(s)+\left(\alpha_{2} / \rho_{2}\right) u_{1}(s)}}{K \rho_{2}} \\
& \quad \cdot \sum_{n=0}^{m_{2}-1} \frac{(-1)^{n}\left(1-m_{2}\right)_{n}}{(n !)^{2}}\left(\frac{\delta_{2}}{\rho_{2}}\right)^{n} \cdot \int_{0}^{\infty}\left(1+\frac{\varepsilon}{w(s)}\right) \\
& \cdot e^{\varepsilon u_{2}(s) / w(s)-\left(\left(\beta_{2}-\delta_{2}\right) / \rho_{2}\right) w(s)}(w(s) \\
& \left.-u_{1}(s)\right)^{n} d w .
\end{aligned}
$$

Then, by using the Binomial expansion for $\left(w(s)-u_{1}(s)\right)^{n}$, we can rewrite (A.11) as

$$
\begin{aligned}
& M_{\gamma_{02}}(s)=\frac{u_{2}(s) \alpha_{2} e^{-K+u_{2}(s)+\left(\alpha_{2} / \rho_{2}\right) u_{1}(s)}}{K \rho_{2}} \\
& \quad \cdot \sum_{n=0}^{m_{2}-1} \frac{(-1)^{n}\left(1-m_{2}\right)_{n}}{(n !)^{2}}\left(\frac{\delta_{2}}{\rho_{2}}\right)^{n} \cdot \sum_{k=0}^{n}\left[-u_{1}(s)\right]^{n-k} \\
& \quad \cdot \int_{0}^{\infty}\left(1+\frac{\varepsilon}{w(s)}\right) \\
& \cdot e^{\varepsilon u_{2}(s) / w(s)-\left(\left(\beta_{2}-\delta_{2}\right) / \rho_{2}\right) w(s)}(w(s))^{k} d w .
\end{aligned}
$$


The integral part of (A.12) can be solved by using [36, Eq. (3.471.9)] as follows:

$$
\begin{aligned}
& \int_{0}^{\infty}\left(1+\frac{\varepsilon}{w(s)}\right) \\
& \cdot e^{\varepsilon u_{2}(s) / w(s)-\left(\left(\beta_{2}-\delta_{2}\right) / \rho_{2}\right) w(s)}(w(s))^{k} d w \\
& \quad=\int_{0}^{\infty} e^{\varepsilon u_{2}(s) / w(s)-\left(\left(\beta_{2}-\delta_{2}\right) / \rho_{2}\right) w(s)}(w(s))^{k} d w \\
& +\varepsilon \int_{0}^{\infty} e^{\varepsilon u_{2}(s) / w(s)-\left(\left(\beta_{2}-\delta_{2}\right) / \rho_{2}\right) w(s)}(w(s))^{k-1} d w \\
& =2\left(\frac{\varepsilon \rho_{2} u_{2}(s)}{\beta_{2}-\delta_{2}}\right)^{(k+1) / 2} \\
& +K_{k+1}\left(2 \sqrt{\frac{\varepsilon u_{2}(s)\left(\beta_{2}-\delta_{2}\right)}{\rho_{2}}}\right) .
\end{aligned}
$$

Therefore, by plugging (A.13) into (A.12), the result of $M_{\gamma_{02}}(s)$ can be obtained as presented in (27).

\section{Conflicts of Interest}

The authors declare no conflicts of interest regarding the publication of this paper.

\section{Authors' Contributions}

Jian Jiao, Houlian Gao, and Qinyu Zhang contributed equally to this work.

\section{Acknowledgments}

This work was supported in part by the National Natural Sciences Foundation of China (NSFC) under Grants 61771158, 61701136, 61525103, and 61371102, the National High Technology Research \& Development Program no. 2014AA01A704, the Natural Scientific Research Innovation Foundation in Harbin Institute of Technology under Grant HIT.NSRIF.2017051, and the Shenzhen Fundamental Research Project under Grants JCYJ20160328163327348 and JCYJ20150930150304185.

\section{References}

[1] N. Zhang, S. Zhang, P. Yang, O. Alhussein, W. Zhuang, and X. S. Shen, "Software Defined Space-Air-Ground Integrated Vehicular Networks: Challenges and Solutions," IEEE Communications Magazine, vol. 55, no. 7, pp. 101-109, 2017.

[2] S. Zhang, N. Zhang, X. Fang, P. Yang, and X. S. Shen, "Self-Sustaining Caching Stations: Toward Cost-Effective 5GEnabled Vehicular Networks," IEEE Communications Magazine, vol. 55, no. 11, pp. 202-208, 2017.
[3] S. Zhang, N. Zhang, P. Yang, and X. S. Shen, "Cost-Effective Cache Deployment in Mobile Heterogeneous Networks," IEEE Transactions on Vehicular Technology, In press.

[4] N. Cheng, H. Zhou, L. Lei et al., "Performance Analysis of Vehicular Device-to-Device Underlay Communication," IEEE Transactions on Vehicular Technology, vol. 66, no. 6, pp. 54095421, 2017.

[5] M. Á. Vázquez, A. Pérez-Neira, D. Christopoulos et al., "Precoding in multibeam satellite communications: Present and future challenges," IEEE Wireless Communications Magazine, vol. 23, no. 6, pp. 88-95, 2016.

[6] S. Maleki, S. Chatzinotas, B. Evans et al., "Cognitive spectrum utilization in Ka band multibeam satellite communications," IEEE Communications Magazine, vol. 53, no. 3, pp. 24-29, 2015.

[7] J. Jiao, X. Sui, S. Gu, S. Wu, and Q. Zhang, "Partially Observable Markov Decision Process-Based Transmission Policy over KaBand Channels for Space Information Networks," Entropy, vol. 19, no. 10, p. 510, 2017.

[8] Y. Feria, M. Belongie, T. Mcpheeters, and H. Tan, "Solar scintillation effects on yelecommunication links at Ka-Band and X-Band," Telecommunications and Data Acquisition Progress Report, vol. 129, pp. 1-11, 1997.

[9] R. F. Arenstorf, "A new method of perturbation theory and its application to the satellite problem of celestial mechanics," Journal für die reine und Angewandte Mathematik, vol. 1966, no. 221, pp. 113-145, 1966.

[10] C. Zhong, S. Jin, and K.-K. Wong, "Dual-hop systems with noisy relay and interference-limited destination," IEEE Transactions on Communications, vol. 58, no. 3, pp. 764-768, 2010.

[11] L. Yang and M. O. Hasna, "Performance Analysis of Amplify-and-Forward Hybrid Satellite-Terrestrial Networks with Cochannel Interference," IEEE Transactions on Communications, vol. 63, no. 12, pp. 5052-5061, 2015.

[12] K. An, M. Lin, T. Liang et al., "Performance Analysis of MultiAntenna Hybrid Satellite-Terrestrial Relay Networks in the Presence of Interference," IEEE Transactions on Communications, vol. 63, no. 11, pp. 4390-4404, 2015.

[13] Y. Wang, J. Jiao, X. D. Sui, S. H. Wu, Y. H. Li, and Q. Y. Zhang, "Rateless coding scheme for time-varying dying channels," in Proceedings of the 8th Wireless Communications Signal Processing (WCSP), Yangzhou, China, October 2016.

[14] Y. Ruan, Y. Li, R. Zhang, and H. Zhang, "Performance analysis of hybrid satellite-terrestrial cooperative networks with distributed alamouti code," in Proceedings of the 83rd IEEE Vehicular Technology Conference (VTC Spring), Nanjing, China, May 2016.

[15] A. Iqbal and K. M. Ahmed, "A hybrid satellite-terrestrial cooperative network over non identically distributed fading channels," Journal of Communications, vol. 6, no. 7, pp. 581-589, 2011.

[16] S. Sreng, B. Escrig, and M.-L. Boucheret, "Exact outage probability of a hybrid satellite terrestrial cooperative system with best relay selection," in Proceedings of the 2013 IEEE International Conference on Communications (ICC), pp. 4520-4524, Hungary, June 2013.

[17] M. R. Bhatnagar and M. K. Arti, "Performance analysis of AF based hybrid satellite-terrestrial cooperative network over generalized fading channels," IEEE Communications Letters, vol. 17, no. 10, pp. 1912-1915, 2013.

[18] V. K. Sakarellos and A. D. Panagopoulos, "Outage performance of cooperative Land Mobile Satellite broadcasting systems," in 
Proceedings of the IEEE European Conference on Antennas and Propagation, pp. 473-476, April 2013.

[19] V. K. Sakarellos, C. Kourogiorgas, and A. D. Panagopoulos, "Cooperative Hybrid Land Mobile Satellite-Terrestrial Broadcasting Systems: Outage Probability Evaluation and Accurate Simulation," Wireless Personal Communications, vol. 79, no. 2, pp. 1471-1481, 2014.

[20] M. Li, M. Lin, Q. Yu, W.-P. Zhu, and L. Dong, "Optimal beamformer design for dual-hop MIMO AF relay networks over rayleigh fading channels," IEEE Journal on Selected Areas in Communications, vol. 30, no. 8, pp. 1402-1414, 2012.

[21] J. Wang, H. Zhu, and N. J. Gomes, "Distributed antenna systems for mobile communications in high speed trains," IEEE Journal on Selected Areas in Communications, vol. 30, no. 4, pp. 675-683, 2012.

[22] H. Zhu, "Performance comparison between distributed antenna and microcellular systems," IEEE Journal on Selected Areas in Communications, vol. 29, no. 6, pp. 1151-1163, 2011.

[23] P.-D. Arapoglou, K. Liolis, M. Bertinelli, A. Panagopoulos, P. Cottis, and R. De Gaudenzi, "MIMO over satellite: a review," IEEE Communications Surveys \& Tutorials, vol. 13, no. 1, pp. 2751, 2011.

[24] A. M.k. and M. R. Bhatnagar, "Beamforming and combining in hybrid satellite-terrestrial cooperative systems," IEEE Communications Letters, vol. 18, no. 3, pp. 483-486, 2014.

[25] N. I. Miridakis, D. D. Vergados, and A. Michalas, "Dual-Hop Communication over a Satellite Relay and Shadowed Rician Channels," IEEE Transactions on Vehicular Technology, vol. 64, no. 9, pp. 4031-4040, 2015.

[26] P. K. Upadhyay and P. K. Sharma, "Max-Max user-relay selection scheme in multiuser and multirelay hybrid satelliteTerrestrial relay systems," IEEE Communications Letters, vol. 20, no. 2, pp. 268-271, 2016.

[27] Y. Dhungana, N. Rajatheva, and C. Tellambura, "Performance analysis of antenna correlation on LMS-Based dual-hop AF MIMO systems," IEEE Transactions on Vehicular Technology, vol. 61, no. 8, pp. 3590-3602, 2012.

[28] Y. Dhungana and N. Rajatheva, "Analysis of LMS based dual hop MIMO systems with beamforming," in Proceedings of the 2011 IEEE International Conference on Communications, Kyoto, Japan, June 2011.

[29] M. R. Bhatnagar, "Performance Evaluation of Decode-andForward Satellite Relaying," IEEE Transactions on Vehicular Technology, vol. 64, no. 10, pp. 4827-4833, 2015.

[30] M. K. Arti and M. R. Bhatnagar, "Two-way mobile satellite relaying: A beamforming and combining based approach," IEEE Communications Letters, vol. 18, no. 7, pp. 1187-1190, 2014.

[31] K. An, J. Ouyang, M. Lin, and T. Liang, "Outage Analysis of Multi-Antenna Cognitive Hybrid Satellite-Terrestrial Relay Networks with Beamforming," IEEE Communications Letters, vol. 19, no. 7, pp. 1157-1160, 2015.

[32] M. Jia, X. Gu, Q. Guo, W. Xiang, and N. Zhang, "Broadband hybrid satellite-terrestrial communication systems based on cognitive radio toward 5G," IEEE Wireless Communications Magazine, vol. 23, no. 6, pp. 96-106, 2016.

[33] F. Lu, X. S. Zhang, and Z. X. Tian, "Analysis of influence on satellite-to-earth data transmission link by directing precision of Ka-band spot beam antenna," Spacecraft Engineering, vol. 25, no. 6, pp. 61-68, 2016.

[34] F. Wang, X. Z. Li, and J. Meng, "Analysis of the impact of elevation on satellite data quality," in Proceedings of the in
Nineteenth Academic conference of Chinese Society of Space Research Detection Professional Committee, 2006.

[35] J. Jiao, Y. Yang, B. Feng, S. Wu, Y. Li, and Q. Zhang, "Distributed Rateless Codes with Unequal Error Protection Property for Space Information Networks," Entropy, vol. 19, no. 1, p. 38, 2017.

[36] A. Jeffrey and D. Zwillinger, Table of Integrals, Series, and Products, Academic Press, 2007.

[37] M. R. Mckay, A. Zanella, I. B. Collings, and M. Chiani, "Error probability and SINR analysis of optimum combining in Rician fading," IEEE Transactions on Communications, vol. 57, no. 3, pp. 676-687, 2009.

[38] S. Gu, J. Jiao, and Q. Zhang, "Intermediate performance of rateless codes over dying erasure channel," in Proceeding of the 6th International Conference on Communications, Signal Processing, and Systems (CSPS2017), Harbin, China, July 2017.

[39] A. Abdi, W. C. Lau, M. Alouini, and M. Kaveh, "A new simple model for land mobile satellite channels: First-and secondorder statistics," IEEE Transactions on Wireless Communications, vol. 2, no. 3, pp. 519-528, 2003.

[40] K. T. Alfriend, H. Schaub, and D.-W. Gim, "Gravitational perturbations, nonlinearity and circular orbit assumption effects on formation flying control strategies," Guidance and Control, pp. 139-155, 2000.

[41] H. Gao, J. Jiao, R. Zhang, S. Gu, S. Wu, and Q. Zhang, "Outage performance of cooperative deep-space downlink with backbone relaying in space-based information networks," in Proceeding of the 6th International Conference on Communications, Signal Processing, and Systems (CSPS2017), Harbin, China, July 2017.

[42] A. F. B. de Almeida Prado, "Third-body perturbation in orbits around natural satellites," Journal of Guidance, Control, and Dynamics, vol. 26, no. 1, pp. 33-40, 2003.

[43] Y. Kozai, "A new method to compute Lunisolar perturbations in satellite motions," Sao Special Report, 1973.

[44] D. Vokrouhlicky, P. Farinella, and F. Mignard, "Solar radiation pressure perturbations for Earth satellites I: A complete theory including penumbra transitions," Astronomy \& Astrophysics, vol. 280, no. 1, pp. 295-312, 1993.

[45] A. Martyusheva, K. Oskina, N. Petrov, and E. Polyakhova, "Solar radiation pressure influence in motion of asteroids, including near-Earth objects," in Proceedings of the 2015 International Conference on Mechanics - Seventh Polyakhov's Reading, Saint Petersburg, Russia, February 2015.

[46] L. M. Buchsbaum, "Pointing losses in single-axis and fixed-mount earth-station antennas due to satellite movement," International Journal of Satellite Communications and Networking, vol. 4, no. 2, pp. 89-96, 1986.

[47] S. Q. Min, Design and Application of Satellite Communication System, Publishing House of Electronics Industry, 2015.

[48] M. Pätzold, Mobile Fading Channels, John Wiley \& Sons, Ltd, Chichester, UK, 2002.

[49] Iskandar and S. Shimamoto, "Channel characterization and performance evaluation of mobile communication employing stratospheric platforms," IEICE Transactions on Communications, vol. E89-B, no. 3, pp. 937-944, 2006.

[50] I. Wolfram, Mathematica Edition: Version 8.0, Wolfram Research Inc, Champaign, Illinois, USA, 2010.

[51] M. Abramowitz and I. A. Stegun, Handbook of mathematical functions: with formulas, graphs, and mathematical tables, Courier Corporation, 1964. 
[52] M. K. Simon and M. Alouini, Digital Communication Over Fading Channels, John Wiley \& Sons, Inc., New York, NY, USA, 2005.

[53] O. Montenbruck and E. Gill, Satellite Orbits: Models, Methods and Applications, Springer Science and Business Media, 2012.

[54] N. Zhengtao, "Progress and present status of research on Earths Gravitational Field," Journal of Geomatics, vol. 1, 2013. 


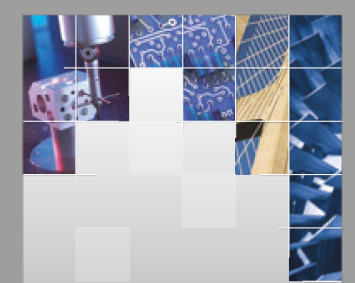

\section{Enfincering}
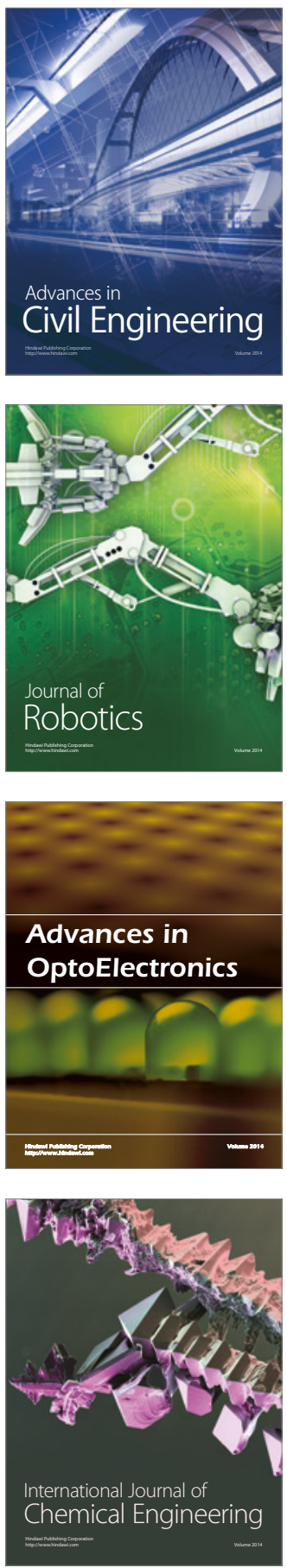

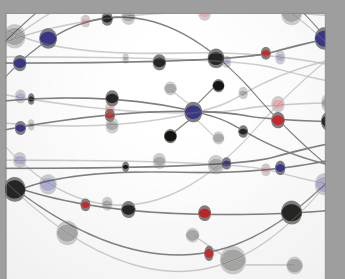

The Scientific World Journal

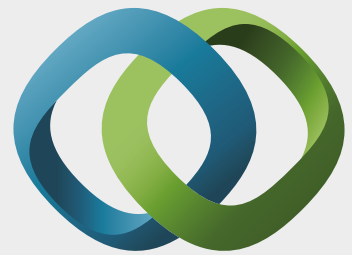

\section{Hindawi}

Submit your manuscripts at

https://www.hindawi.com
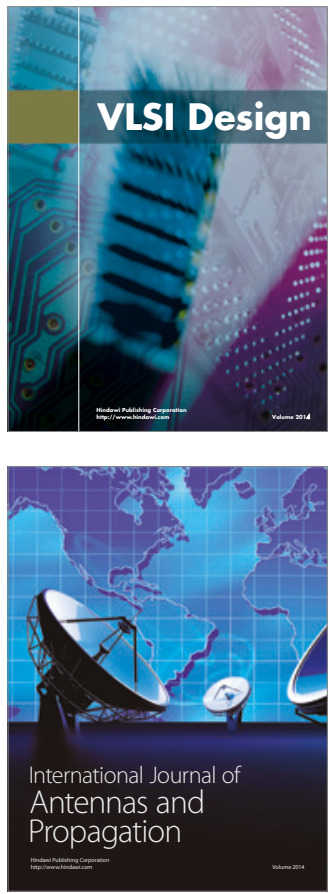

\section{Rotating}

Machinery
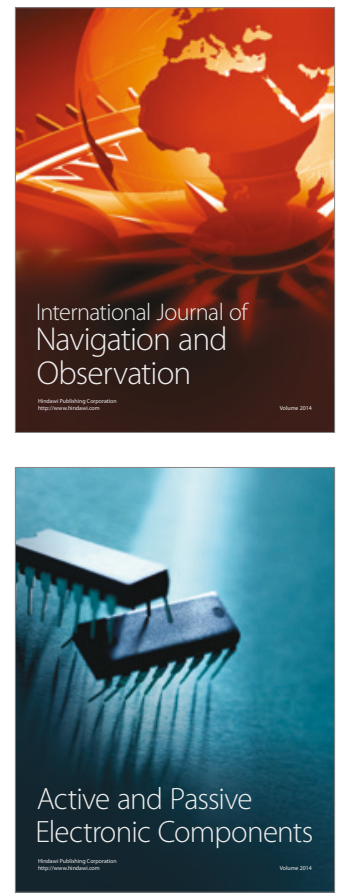
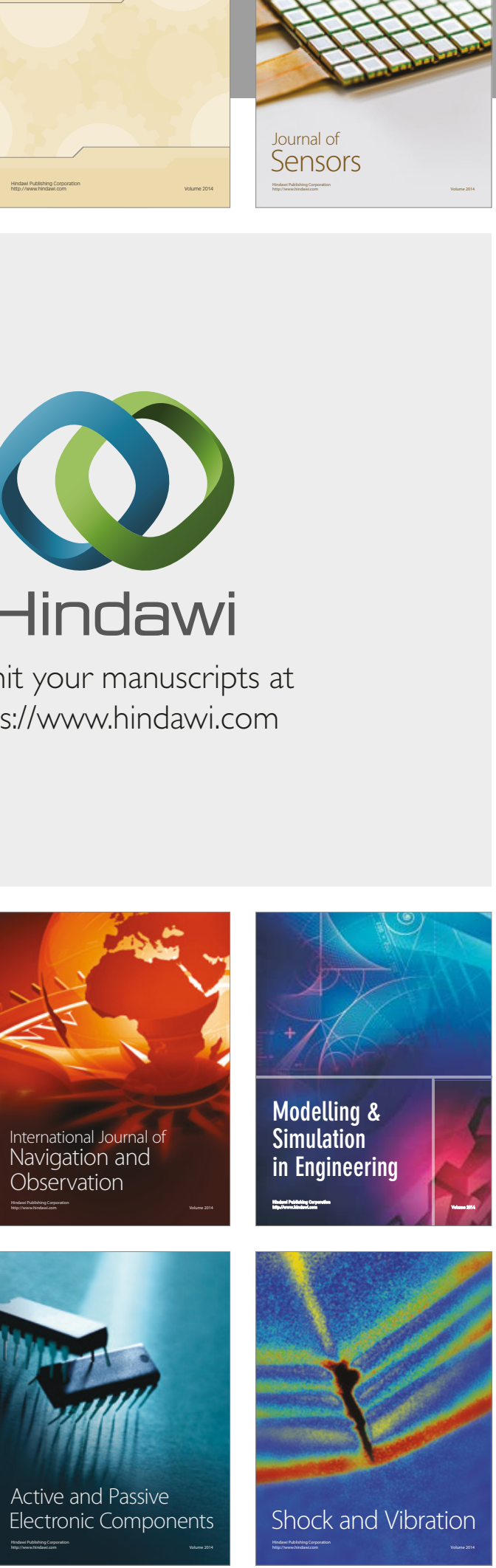
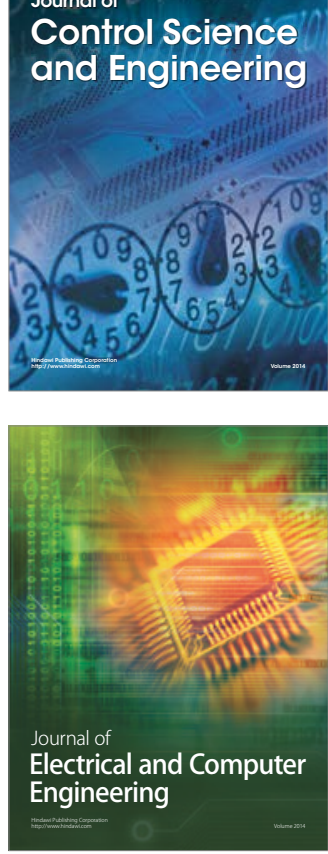

Distributed

Journal of

Control Science

and Engineering
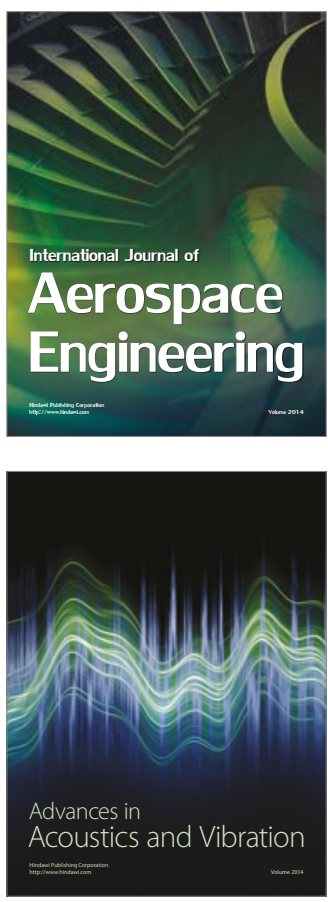

Sensor Networks 